\title{
Adult Ear Tubes
}

\author{
Dennis C Fitzgerald*, Thomas Wilcox and Rebecca Chiffer \\ Department of Otolaryngology-Head and Neck Surgery, Jefferson Balance and Hearing Center, USA.
}

*Corresponding author: Dennis C Fitzgerald, Department of Otolaryngology-Head and Neck Surgery, Jefferson Balance and Hearing Center, Thomas Jefferson University, USA.

Received Date: August 20, 2020

Published Date: September 04, 2020

\section{Short Communication}

Ear tubes, a.k.a. PE tubes are one of the most common operative procedures performed in the USA. Most tubes are placed in children for recurrent ear infections and serous otitis media (middle ear fluid). It is estimated that over 500,000 tubes are placed each year. Most of the patient information available relates to the placement of these tubes in children. Little is available for tubes paced in adults.

\section{Myringotomy or ear tubes in adults}

When medical therapy is ineffective for the treatment of chronic ear fluid, recurrent ear infections, and hearing loss, myringotomy or ear tubes (PE tubes) may be recommended. These treatments may also be recommended for problems with pressure or fullness of the ears, This fullness usually occurs from allergies or exposure to altitude changes. Less common causes of ear fullness include temporomandibular disorder (TMD), migraine, and endolymphatic hydrops. These causes of fullness would not be appropriate reasons for myringotomy or ear tubes. When a surgical intervention is indicated, the physician may recommend only a myringotomy. This means that an opening is made in the eardrum, but a tube is NOT placed into the eardrum. The eardrum usually heals up on its own after a few days. The procedure is usually done in the office, by using a local anesthetic to numb the eardrum.

To insert an ear tube, a same small incision is made in the eardrum, similar to a myringotomy, which allows the drainage of ear fluid and for air to enter the middle ear and re-establish an aerated middle ear. Hearing is almost immediately restored. Local anesthesia is the same as for a myringotomy. Most adults are able to have this done in the office. Sometimes, due to anxiety or anatomic abnormalities, it will be done in the operating room.
Tympanostomy tubes usually stay in place from 9-18 months and fall out of the eardrum.

The tubes are tiny and are not visible. Initially, there may be a sensation of hearing your own voice and/or pressure, but this usually goes away with time. Ear tubes reduce the severity and frequency of infections, development of middle ear fluid, and conductive hearing loss. However, they cannot reverse the underlying reasons for the ear disease (allergies, eustachian tube dysfunction etc.).

The ear tubes work by allowing air to enter into the middle ear space and drainage of the fluid behind the eardrum. This improves the function of the middle ear (the cavity behind the eardrum). The tubes replace the function of the Eustachian tube whose function is to allow air to intermittently but regularly enter the middle ear space. Placement of ear tubes has few risks. When the tubes are in place, it is not unusual for patients to have drainage when they get "colds" or allergy symptoms. When the tubes fall out, patients rarely will have a small hole in the eardrum (perforation) that could require additional surgical repair.

\section{Instructions after the procedure}

It is not unusual to have ear drainage for a couple of days after surgery. Sometimes this drainage can be

bloody, which can be a sign of inflammation. If you are prescribed ear drops, or sometimes eye drops, use them as directed. Mild ear pain on the night of insertion may require treatment with Tylenol. Normal activity can usually be resumed the same day of the procedure. Most people are able to drive home from the office. 
Water should be kept out of the ears for as long as the tubes are in place. This can be done by placing a cotton ball covered with Vaseline Petroleum jelly in the ear canal. Usually earplugs are needed during swimming and other water exposure while the tubes are in place. Avoid the use of Q-tips. Please keep your appointment as scheduled or be sure to call back to make follow-up appointments as directed by physician.

\section{Acknowledgement}

None.

\section{Conflict of Interest}

No conflict of interest. 